Mathematical Modelling and Analysis

Volume 21 Number 4, July 2016, 431-449

http://dx.doi.org/10.3846/13926292.2016.1175388

(c) Vilnius Gediminas Technical University, 2016
Publisher: Taylor\&Francis and VGTU

http://www.tandfonline.com/TMMA

ISSN: $1392-6292$

eISSN: 1648-3510

\title{
Anisotropic Quasi-Wilson Element with Conforming Finite Element Approximation for Coupled Continuum Pipe-Flow/Darcy Model in Karst Aquifers
}

\section{Wei Liu ${ }^{a}$ and Jintao $\mathrm{Cui}^{b}$}

\author{
${ }^{a}$ School of Mathematics and Statistics Science, Ludong University \\ 264025 Yantai, China \\ ${ }^{b}$ Department of Applied Mathematics, The Hong Kong Polytechnic University \\ Kowloon, Hong Kong \\ E-mail(corresp.): sduliuwei@mail.sdu.edu.cn
}

Received May 10, 2015; revised April 1, 2016; published online July 1, 2016

\begin{abstract}
This paper presents a numerical method for solving systems of partial differential equations describing flow in porous media with an embedded and inclined conduit pipe. This work considers a coupled continuum pipe-flow/Darcy model. The numerical schemes presented are based on combinations of the quasi-Wilson element on anisotropic mesh and the conforming finite element on regular mesh. The existence and uniqueness of the approximation solution are obtained. Optimal error estimates in both $L^{2}$ and $H^{1}$ norms are obtained independent of the regularity condition on the mesh. Numerical examples show the accuracy and efficiency of the proposed scheme.
\end{abstract}

Keywords: Karst aquifers, quasi-Wilson element, anisotropic mesh, coupled continuum pipe-flow/Darcy model, numerical analysis.

AMS Subject Classification: 65N15; 65N30.

\section{Introduction}

Managing the quality of groundwater resources is imperative today with the risks of environmental and industrial contamination. Therefore the development and study of numerical models of groundwater flow in Karst aquifers, which are very vulnerable sources of groundwater, is a most practical application. One of the most popular models is the coupled continuum pipeflow/Darcy (CCPF) model in which the conduits embedded in the continuum matrix are simplified into a network of one-dimensional pipes, see $[2,3,4,5,11$, 19]. As shown in Figure 1, the conduit $\Omega^{c}$ in the model of a Karst aquifer is a one-dimensional curve with sinkhole boundary $\Gamma_{s i}$ and $\Gamma_{s p}$, which are the end points of the curve. 


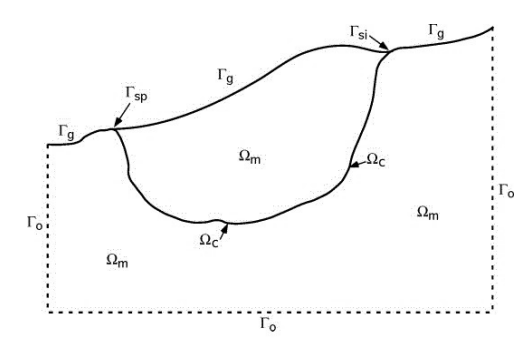

Figure 1. Two-dimensional figure of a Karst aquifer.

The continuum $\Omega^{m}$ denotes the domain occupied by the porous media around the curve. $\Gamma_{g}$ represents the ground surface boundary, and $\Gamma_{0}$ shows a bounding surface that is presumably far removed from the region of interest.

In general, the flow in the porous matrix is modeled by a continuum approach using the Darcy equation $[5,14]$, and one-dimensional pipe-flow equation [5] is applied to the conduit flow in the tube. In order to preserve the conservation of mass, the matrix flow and conduit flow are coupled at the interface by the exchange flux, which is determined linearly by the difference of hydraulic heads between the matrix system and the conduit system. See, e.g., Cao et al. [5], Lei [10], Chen [9] and Wu [20].

A simplified sketch is shown in Figure 2.

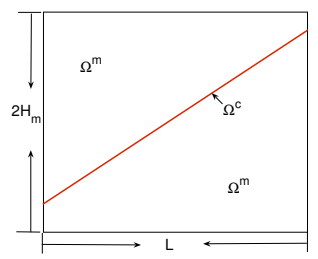

Figure 2. A simplified sketch of a Karst aquifer.

We consider the two-dimensional porous domain $\Omega^{m}=(0, L) \times\left(-H_{m}, H_{m}\right)$, and the inclined conduit pipe $\Omega^{c}=\{y=k x+b, x \in(0, L)\}$ with real numbers $k$ and $b$, where $2 H_{m}$ is the height of the matrix, $L$ is the horizontal length of the matrix. We assume $k L+b<H_{m}$ and $b>-H_{m}$, so that the inclined conduit is embedded in the porous media. In conclusion, the steady-state coupled continuum pipe-flow/Darcy (CCPF) model in Karst aquifers is given as follows.

$$
\begin{cases}-\nabla \cdot\left(\mathbb{K} \nabla h^{m}\right)=-\alpha_{e x}\left(h^{m}-h^{c}\right) \delta_{\Omega^{c}}+f^{m}, & \text { in } \Omega^{m}, \\ -\frac{\partial}{\partial \tau}\left(D \frac{\partial h^{c}}{\partial \tau}\right)=\alpha_{e x}\left(\left.h^{m}\right|_{\Omega^{c}}-h^{c}\right)+f^{c}, & \text { in } \Omega^{c},\end{cases}
$$

where $h^{m}$ and $h^{c}$ denote the unknown hydraulic heads in the porous matrix 
$\Omega^{m}$ and conduit pipe $\Omega^{c}$, respectively.

Under the homogeneous isotropic media assumption, the hydraulic conductivity tensor $\mathbb{K}$ takes the form of $\mathbb{K}=\mathcal{K} \mathbb{I}$. Here $\mathcal{K}$ is a constant, $\mathcal{K}=\frac{\tilde{k} g}{\mu}$, where $\tilde{k}$ is the constant matrix permeability, $\mu$ the kinematic viscosity of water, and $g$ the gravitational acceleration constant. We use $\tau$ to express the unit tangential direction along one-dimensional inclined pipe conduit. The conductivity constant $D$ depends on the width of the conduit $d$ such that $D=\frac{d^{3} g}{12 \mu} ; f^{m}$ and $f^{c}$ represent the external source or sink terms; $\delta_{\Omega^{c}}$ is the Dirac delta function focused on $\Omega^{c}$. The nonnegative constant $\alpha_{e x}$ represents the coefficient of flux exchange at the intersection between the matrix and conduit flow. Physical experimental results in Chen et al. [6] show that the CCPF model is valid for flows in Karst aquifers when a suitable fluid exchange coefficient $\alpha_{e x}$ is taken.

A suitable boundary condition is needed to solve the coupled model (1.1). We suppose $h^{c}$ is specified at the end points of the conduit, $\Gamma_{s i}$ and $\Gamma_{s p}$, and $h^{m}$ is specified on the boundary of $\Omega^{m}$ given by $\Gamma_{g} \cup \Gamma_{0}$.

The time-dependent case of (1.1) first appeared in [2,3,4], where Karst aquifer genesis is studied. Combining the idea of dual porosity, the model (1.1) has been discussed by the Carbonate Aquifer Void Evolution (CAVE) code, which solved the flow model in the porous matrix by a finite difference scheme using MODFLOW and the flow model in conduit by a nonlinear finite difference discretization. Wang [19] demonstrated that the coupled continuum pipe-flow/Darcy model (1.1) is well-posed in two spatial dimensions. Cao et al. [5] applied conforming finite element approximation to solve the CCPF model with a horizontal pipe cube $\Omega^{c}=\{y=0\}$, and obtained optimal convergence rates in the $L^{2}$ and $H^{1}$ norms based on regular grid. Liu et al. used anisotropic finite element method and coupled finite element method to solve the same model as in [5] based on the horizontal pipe domain in [12] and [13], respectively.

The objective of this paper is to study CCPF model with the inclined pipe cube embedded in the porous media. Considering the large angle of inclination of the conduit pipe, the rectangular grid and even the regular mesh can not be employed on the solved two-dimensional region. Therefore, we shall choose the arbitrary quadrilateral grids as the regional division to approximate the Darcy equation of CCPF model. In order to avoid the limits on the subdivision, the anisotropic quadrilateral mesh is considered without the regularity condition. The second goal of this paper is to introduce the quasi-Wilson element to approximate the two-dimensional Darcy equation of CCPF model (1.1) and conforming finite element to solve the pipe-flow equation. As is known to all, the quasi-Wilson element is a nonconforming element on quadrilateral mesh. The element comes from Shi-Chen $[17,18]$ which simply adds a term of high order to the nonconforming part of the shape basis functions. In recent years, there have been many research papers such as $[7,8,15,16]$ with respect to numerical analysis of quasi-Wilson element.

Herein, the existence and uniqueness of the solution are obtained for the coupled approximation scheme based on the quasi-Wilson nonconforming finite element method for Darcy equation with conforming finite element method for pipe-flow equation. The optimal error estimates in both $L^{2}$ and $H^{1}$ norms are established independent of the regularity condition on the mesh. Numerical 
results, based on two different anisotropic subdivisions, show the efficiency and accuracy of our scheme.

The outline of the paper is as follows. The anisotropic mesh of $\Omega^{m}$ and the regular mesh on the domain $\Omega^{c}$ are presented in Section 2. The approximation scheme using quasi-Wilson nonconforming element on anisotropic grid combined with conforming finite element method is carried out for the coupled CCPF model. Existence and uniqueness of approximation solution are also deduced in Section 2. Section 3 gives the error estimates of the approximation scheme based on the anisotropic interpolation properties. Numerical examples are given to show the efficiency of the scheme in Section 4, followed by conclusions.

Throughout this paper, we use $C, C_{1}$ and $C_{2}$ to denote generic positive constants independent of the discretization parameters, which may have different values in different appearances.

The Sobolev space $H^{m}(\otimes)=W^{m, 2}(\otimes)$ is defined in the usual ways with the usual norm $\|\cdot\|_{m, \otimes}$ and semi-norm $|\cdot|_{m, \otimes}$, where $\otimes$ is $\Omega^{c}$ or $\Omega^{m}$ or some other domains.

\section{Quasi-Wilson nonconforming element with conforming finite element method}

In this section we present the finite element scheme for the coupled problem. We use the quasi-Wilson element based on anisotropic mesh for $h^{m}$. We also present the existence and uniqueness of the approximation solution.

It follows from property of Dirac delta function and a technique of coordinate transformation that

$$
\int_{0}^{L} \int_{-H_{m}}^{H_{m}} f(x, y) \delta(y-(k x+b)) d x d y=\int_{\Omega^{c}} f(x, k x+b) d s .
$$

Then, the weak form of the simplified CCPF model (1.1) with zero Dirichlet boundary condition is to find $\mathbf{h}=\left(h^{m}, h^{c}\right) \in H_{0}^{1}\left(\Omega^{m}\right) \times H_{0}^{1}\left(\Omega^{c}\right)$ such that

$$
a(\mathbf{h}, \mathbf{v})=(\mathbf{f}, \mathbf{v}), \quad \forall \mathbf{v}=\left(v^{m}, v^{c}\right) \in H_{0}^{1}\left(\Omega^{m}\right) \times H_{0}^{1}\left(\Omega^{c}\right),
$$

where the bilinear form

$$
\begin{aligned}
a(\mathbf{h}, \mathbf{v})= & \int_{\Omega^{m}} \mathbb{K} \nabla h^{m} \nabla v^{m} d x d y+\int_{\Omega^{c}} D \frac{\partial h^{c}}{\partial \tau} \frac{\partial v^{c}}{\partial \tau} d s \\
& +\left.\alpha_{e x} \int_{\Omega^{c}}\left(\left.h^{m}\right|_{\Omega^{c}}-h^{c}\right) v^{m}\right|_{\Omega^{c}} d s-\alpha_{e x} \int_{\Omega^{c}}\left(\left.h^{m}\right|_{\Omega^{c}}-h^{c}\right) v^{c} d s
\end{aligned}
$$

and

$$
(\mathbf{f}, \mathbf{v})=\int_{\Omega^{m}} f^{m} v^{m} d x d y+\int_{\Omega^{c}} f^{c} v^{c} d s
$$

with $\mathbf{f}=\left(f^{m}, f^{c}\right)$. 
Lemma 1. The weak solution $\mathbf{h}$ of simplified CCPF model (1.1) exists and is unique.

Proof. According to [5], this is a straight application of Lax-Milgram Theorem on the fact that the bilinear form $a(\mathbf{h}, \mathbf{v})$ on $\left(H_{0}^{1}\left(\Omega^{m}\right) \times H_{0}^{1}\left(\Omega^{c}\right)\right) \times\left(H_{0}^{1}\left(\Omega^{m}\right) \times\right.$ $\left.H_{0}^{1}\left(\Omega^{c}\right)\right)$ satisfies the continuity and coercivity conditions.

The domain $\Omega^{m}$ is subdivided into convex quadrilateral triangulation $\mathcal{T}_{h}=$ $\{K\}$, where $K$ is a quadrilateral element. Since the solution $h^{m}$ varies significantly near the pipe region $\Omega^{c}=\{y=k x+b, x \in(0, L)\}$, we consider to use anisotropic meshes with a small mesh size near the conduit region and a larger mesh size elsewhere. For example, we shall employ one subdivision way with nonuniform grid along the changes of position of $\Omega^{c}$ and uniform grid on $x$-direction. The subdivision of $\Omega^{c}$ is obtained by $\mathcal{T}_{h}$ restriction on the inclined conduit pipe. Therefore, the domain $\Omega^{m}$ is occupied by anisotropic mesh and the domain $\Omega^{c}=\{I\}$ is covered with regular cells.

Suppose $K$ is a convex quadrilateral element with four vertices $p_{1}\left(x_{1}, y_{1}\right)$, $p_{2}\left(x_{2}, y_{2}\right), p_{3}\left(x_{2}, y_{3}\right)$ and $p_{4}\left(x_{1}, y_{4}\right)$. Denote

$$
h_{x, K}=\frac{1}{2} \max \left\{\left|p_{1} p_{2}\right|,\left|p_{3} p_{4}\right|\right\}, \quad h_{y, K}=\frac{1}{2} \max \left\{\left|p_{2} p_{3}\right|,\left|p_{4} p_{1}\right|\right\} .
$$

Define $h_{K}=\max \left\{h_{x, K}, h_{y, K}\right\}$ and $h=\max _{K \in \mathcal{T}_{h}}\left\{h_{K}\right\}$. Let $\rho_{K}$ be the supremum of the diameters of all circles contained in element $K$. It's well known that the classical finite element theory relies on the regular condition

$$
\frac{h_{K}}{\rho_{K}} \leq C, \quad \text { for any an element } K,
$$

where $C$ is a positive constant independent of $K$.

At present we do not use the condition (2.2) on the anisotropic meshes $\mathcal{T}_{h}$. We apply the nonconforming quasi-Wilson element based on the anisotropic meshes $\mathcal{T}_{h}$ to solve the first equation of CCPF model (1.1). For this purpose first we present the quasi-Wilson element $\left(\hat{K}, \hat{P}, \hat{\sum}\right)$ on the reference rectangular $\hat{K}$ defined on $(\hat{x}, \hat{y})$-plane with four vertices $\hat{p}_{1}(-1,-1), \hat{p}_{2}(1,-1), \hat{p}_{3}(1,1)$, $\hat{p}_{4}(-1,1)$, and

$$
\begin{aligned}
& \hat{P}=\operatorname{span}\left\{\hat{N}_{i}(\hat{x}, \hat{y}), i=1, \cdots, 6\right\} \\
& \hat{\sum}=\left\{\hat{v}_{i}, i=1, \cdots, 4 ; \frac{\partial^{2} \hat{v}}{\partial \hat{x}^{2}}(0,0), \frac{\partial^{2} \hat{v}}{\partial \hat{y}^{2}}(0,0)\right\},
\end{aligned}
$$

where

$$
\begin{aligned}
& \hat{N}_{1}=\frac{(1-\hat{x})(1-\hat{y})}{4}, \quad \hat{N}_{2}=\frac{(1+\hat{x})(1-\hat{y})}{4}, \quad \hat{N}_{3}=\frac{(1+\hat{x})(1+\hat{y})}{4}, \\
& \hat{N}_{4}=\frac{(1-\hat{x})(1+\hat{y})}{4}, \hat{N}_{5}=\frac{1}{2}\left(\hat{x}^{2}-1\right)-\frac{5}{12}\left(\hat{x}^{4}-1\right), \hat{N}_{6}=\frac{1}{2}\left(\hat{y}^{2}-1\right)-\frac{5}{12}\left(\hat{y}^{4}-1\right),
\end{aligned}
$$

and $\hat{v}_{i}=\hat{v}\left(\hat{p}_{i}\right),(i=1, \cdots, 4)$. Then, any $\hat{v} \in \hat{P}$ can be rewritten as follows

$$
\hat{v}(\hat{x}, \hat{y})=\sum_{i=1}^{4} \hat{N}_{i}(\hat{x}, \hat{y}) \hat{v}_{i}+\hat{N}_{5}(\hat{x}, \hat{y}) \frac{\partial^{2} \hat{v}}{\partial \hat{x}^{2}}(0,0)+\hat{N}_{6}(\hat{x}, \hat{y}) \frac{\partial^{2} \hat{v}}{\partial \hat{y}^{2}}(0,0) \text {. }
$$


Note that there are other choices for $\hat{N}_{5}(\hat{x}, \hat{y})$ and $\hat{N}_{6}(\hat{x}, \hat{y})$, for details see $[7,8,15,16]$.

Let $F_{K}$ be the affine mapping from $\hat{K}$ to $K$ as follows,

$$
\left\{\begin{array}{l}
x=x_{1}\left(\hat{N}_{1}+\hat{N}_{4}\right)+x_{2}\left(\hat{N}_{2}+\hat{N}_{3}\right), \\
y=y_{1} \hat{N}_{1}+y_{2} \hat{N}_{2}+y_{3} \hat{N}_{3}+y_{4} \hat{N}_{4} .
\end{array}\right.
$$

Then,

$$
\begin{array}{ll}
\frac{\partial x}{\partial \hat{x}}=\frac{x_{2}-x_{1}}{2}, & \frac{\partial y}{\partial \hat{x}}=\frac{y_{3}-y_{4}+y_{2}-y_{1}}{4}+\frac{\left(y_{1}-y_{2}+y_{3}-y_{4}\right) \hat{y}}{4}, \\
\frac{\partial x}{\partial \hat{y}}=0, & \frac{\partial y}{\partial \hat{y}}=\frac{y_{3}+y_{4}-y_{2}-y_{1}}{4}+\frac{\left(y_{1}-y_{2}+y_{3}-y_{4}\right) \hat{x}}{4} .
\end{array}
$$

Denote $J_{K}$ be the Jacobi matrix of $F_{K}$. According to simple calculations [8], we obtain that

$$
\begin{aligned}
& \left|\frac{\partial x}{\partial \hat{x}}\right| \leq C h_{x, K}, \quad\left|\frac{\partial y}{\partial \hat{x}}\right| \leq C h_{y, K} \quad\left|\frac{\partial y}{\partial \hat{y}}\right| \leq C h_{y, K}, \\
& \left|\frac{\partial \hat{x}}{\partial x}\right| \leq C h_{x, K}^{-1}, \quad\left|\frac{\partial \hat{x}}{\partial y}\right| \leq C h_{x, K}^{-1} \quad\left|\frac{\partial \hat{y}}{\partial y}\right| \leq C h_{y, K}^{-1} \\
& C h_{x, K} h_{y, K} \leq\left|J_{K}\right| \leq h_{x, K} h_{y, K} .
\end{aligned}
$$

Based on the anisotropic subdivision $\mathcal{T}_{h}$, the finite element space $V_{h}$ is defined by

$$
\begin{gathered}
V_{h}=\left\{v_{h} \in L^{2}\left(\Omega^{m}\right):\left.v_{h}\right|_{K} \text { has the form of } \hat{v}(\hat{x}, \hat{y}) \circ F_{K}^{-1}, \forall K \in \mathcal{T}_{h} ;\right. \\
\left.v_{h} \text { vanishing at the vertices on the boundary of } \Omega^{m}\right\} .
\end{gathered}
$$

For the second equation of CCPF model (1.1), we use conforming finite element approximation based on the partition of $\Omega^{c}$, where the finite element space $W_{h} \subset H_{0}^{1}\left(\Omega^{c}\right)$ consists of continuous piecewise linear polynomials. The approximation scheme of (2.1) based on quasi-Wilson nonconforming element combining with conforming finite element method is in the following form. Find $\mathbf{h}_{h}=\left(h_{h}^{m}, h_{h}^{c}\right) \in V_{h} \times W_{h}$ such that

$$
a_{h}\left(\mathbf{h}_{h}, \mathbf{v}_{h}\right)=\left(\mathbf{f}, \mathbf{v}_{h}\right), \quad \forall \mathbf{v}_{h}=\left(v_{h}^{m}, v_{h}^{c}\right) \in V_{h} \times W_{h},
$$

where the bilinear form

$$
\begin{aligned}
& a_{h}\left(\mathbf{h}_{h}, \mathbf{v}_{h}\right)=\sum_{K \in \mathcal{T}_{h}} \int_{K} \mathbb{K} \nabla h_{h}^{m} \nabla v_{h}^{m} d x d y+\int_{\Omega^{c}} D \frac{\partial h_{h}^{c}}{\partial \tau} \frac{\partial v_{h}^{c}}{\partial \tau} d s \\
& \quad+\left.\alpha_{e x} \int_{\Omega^{c}}\left(\left.\left\{h_{h}^{m}\right\}\right|_{\Omega^{c}}-h_{h}^{c}\right)\left\{v_{h}^{m}\right\}\right|_{\Omega^{c}} d s-\alpha_{e x} \int_{\Omega^{c}}\left(\left.\left\{h_{h}^{m}\right\}\right|_{\Omega^{c}}-h_{h}^{c}\right) v_{h}^{c} d s .
\end{aligned}
$$

Here $\left.\left\{h_{h}^{m}\right\}\right|_{\Omega^{c}}=\frac{\left.h_{h}^{m}\right|_{\Omega_{+}^{c}}+\left.h_{h}^{m}\right|_{\Omega_{-}^{c}}}{2}$ with

$$
\left.h_{h}^{m}\right|_{\Omega_{+}^{c}}=\lim _{y \rightarrow(k x+b)^{+}} h_{h}^{m}(x, y), \quad \text { and }\left.\quad h_{h}^{m}\right|_{\Omega_{-}^{c}}=\lim _{y \rightarrow(k x+b)^{-}} h_{h}^{m}(x, y) .
$$


For any $\mathbf{v}_{h}=\left(v_{h}^{m}, v_{h}^{c}\right) \in V_{h} \times W_{h}$, we introduce a norm given by

$$
\left\|\mathbf{v}_{h}\right\|_{1, h}=\left\|v_{h}^{m}\right\|_{1, h}+\left\|v_{h}^{c}\right\|_{1, \Omega^{c}}=\left(\sum_{K \in \mathcal{T}_{h}}\left|v_{h}^{m}\right|_{1, K}^{2}\right)^{1 / 2}+\left\|v_{h}^{c}\right\|_{1, \Omega^{c}} .
$$

Dividing an element $v_{h}^{m} \in V_{h}$ into two parts, we have $v_{h}^{m}=\widetilde{v_{h}^{m}}+\overline{v_{h}^{m}}$, where $\widetilde{v_{h}^{m}}$ is the conforming part defined by

$$
\left.\widetilde{v_{h}^{m}}\right|_{K}=\widetilde{\hat{v}_{h}^{m}} \circ F_{K}^{-1}=\left(\sum_{i=1}^{4} \hat{N}_{i}(\hat{x}, \hat{y}) \hat{v}_{h}^{m}\left(\hat{p}_{i}\right)\right) \circ F_{K}^{-1}, \quad \forall K \in \mathcal{T}_{h} .
$$

And, $\overline{v_{h}^{m}}$ is the nonconforming part given by

$$
\left.\overline{v_{h}^{m}}\right|_{K}=\overline{\hat{v}_{h}^{m}} \circ F_{K}^{-1}=\left(\hat{N}_{5}(\hat{x}, \hat{y}) f_{1}+\hat{N}_{6}(\hat{x}, \hat{y}) f_{2}\right) \circ F_{K}^{-1}
$$

where $f_{1}=\frac{\partial^{2} \hat{v}}{\partial \hat{x}^{2}}(0,0), f_{2}=\frac{\partial^{2} \hat{v}}{\partial \hat{y}^{2}}(0,0)$.

By the definition (2.8), we see that $\frac{\partial \overline{\hat{v}_{h}^{m}}}{\partial \hat{x}}$ is odd function about $\hat{x}$ and $\frac{\partial \overline{\hat{v}}_{h}^{m}}{\partial \hat{y}}$ is odd function about $\hat{y}$. Combining with the bilinear properties of $\hat{N}_{i}(i=$ $1, \cdots, 4)$, we obtain the following properties.

$$
\int_{\hat{K}} \frac{\partial \widetilde{\hat{v}_{h}^{m}}}{\partial \hat{x}} \frac{\partial \hat{\hat{v}}_{h}^{m}}{\partial \hat{x}} d \hat{x} d \hat{y}=\int_{\hat{K}} \frac{\partial \widetilde{\hat{v}_{h}^{m}}}{\partial \hat{y}} \frac{\partial \overline{\hat{v}_{h}^{m}}}{\partial \hat{y}} d \hat{x} d \hat{y}=0 .
$$

Thus,

$$
\begin{aligned}
\left|\hat{v}_{h}^{m}\right|_{1, \hat{K}}^{2} & =\left\|\frac{\partial \overline{\hat{v}_{h}^{m}}}{\partial \hat{x}}\right\|_{0, \hat{K}}^{2}+\left\|\frac{\partial \overline{\hat{v}_{h}^{m}}}{\partial \hat{y}}\right\|_{0, \hat{K}}^{2}+\left\|\frac{\partial \widetilde{\hat{v}_{h}^{m}}}{\partial \hat{x}}\right\|_{0, \hat{K}}^{2}+\left\|\frac{\partial \widetilde{\hat{v}_{h}^{m}}}{\partial \hat{y}}\right\|_{0, \hat{K}}^{2} \\
& =\left|\widetilde{\hat{v}_{h}^{m}}\right|_{1, \hat{K}}^{2}+\left|\widetilde{\hat{v}_{h}^{m}}\right|_{1, \hat{K}}^{2} .
\end{aligned}
$$

Moreover, we have, by the definition of $\overline{\hat{v}}_{h}^{m}$ in (2.8), that,

$$
\left|\overline{\hat{v}_{h}^{m}}\right|_{1, \hat{K}}^{2}=\frac{16\left(f_{1}^{2}+f_{2}^{2}\right)}{65} \quad \text { and } \quad\left\|\overline{\hat{v}}_{h}^{m}\right\|_{0, \hat{K}}^{2}=\frac{32\left(f_{1}^{2}+f_{2}^{2}\right)}{2835}
$$

then,

$$
\left\|\overline{\hat{v}_{h}^{m}}\right\|_{0, \hat{K}} \leq\left|\overline{\hat{v}_{h}^{m}}\right|_{1, \hat{K}}
$$

Theorem 1. There exists a unique solution $\mathbf{h}_{h}=\left(h_{h}^{m}, h_{h}^{c}\right) \in V_{h} \times W_{h}$ satisfying the approximation scheme (2.5).

Proof. Noticing $W_{h} \subset H_{0}^{1}\left(\Omega^{c}\right)$, the assumptions on hydraulic conductivity tensor $\mathbb{K}$ and the constant $D$, we get for any $\mathbf{v}_{h}=\left(v_{h}^{m}, v_{h}^{c}\right) \in V_{h} \times W_{h}$,

$$
a_{h}\left(\mathbf{v}_{h}, \mathbf{v}_{h}\right)=\sum_{K \in \mathcal{T}_{h}} \int_{K} \mathbb{K} \nabla v_{h}^{m} \nabla v_{h}^{m} d x d y+\int_{\Omega^{c}} D \frac{\partial v_{h}^{c}}{\partial \tau} \frac{\partial v_{h}^{c}}{\partial \tau} d s
$$




$$
\begin{aligned}
& +\alpha_{e x} \int_{\Omega^{c}}\left(\left.\left\{v_{h}^{m}\right\}\right|_{\Omega^{c}}-v_{h}^{c}\right)^{2} d s \geq C\left(\sum_{K \in \mathcal{T}_{h}}\left|v_{h}^{m}\right|_{1, K}^{2}+\left|v_{h}^{c}\right|_{1, \Omega^{c}}^{2}\right) \\
& \geq C\left(\sum_{K \in \mathcal{T}_{h}}\left|v_{h}^{m}\right|_{1, K}^{2}+\left\|v_{h}^{c}\right\|_{1, \Omega^{c}}^{2}\right) \geq C_{1}\left\|\mathbf{v}_{h}\right\|_{1, h}^{2} .
\end{aligned}
$$

Therefore, the approximation bilinear form (2.6) is uniformly $V_{h} \times W_{h}$ elliptic.

By (2.10) and technique of coordinate transformation (2.3)-(2.4),

$$
\begin{aligned}
\sum_{e \in \partial K} \int_{e}\left|\overline{v_{h}^{m}}\right|^{2} d s= & \int_{-1}^{1}\left|\overline{\hat{v}_{h}^{m}}(\hat{x},-1)\right|^{2} \frac{d x}{d \hat{x}} d \hat{x}+\int_{-1}^{1}\left|\overline{\hat{v}_{h}^{m}}(1, \hat{y})\right|^{2} \frac{d y}{d \hat{y}} d \hat{y} \\
& +\int_{-1}^{1}\left|\overline{\hat{v}_{h}^{m}}(\hat{x}, 1)\right|^{2} \frac{d x}{d \hat{x}} d \hat{x}+\int_{-1}^{1}\left|\overline{\hat{v}_{h}^{m}}(-1, \hat{y})\right|^{2} \frac{d y}{d \hat{y}} d \hat{y} \\
\leq & C h_{K}\left\|\overline{\hat{v}_{h}^{m}}\right\|_{0, \hat{K}}^{2} \leq C h_{K}\left|\overline{\hat{v}_{h}^{m}}\right|_{1, \hat{K}}^{2} \\
= & C h_{K} \sum_{|\beta|=1}\left\|\hat{D}^{\beta} \overline{\hat{v}}_{h}^{m}\right\|_{0, \hat{K}}^{2} \\
\leq & C \sum_{|\beta|=1}\left\|D^{\beta} \overline{v_{h}^{m}}\right\|_{0, K}^{2}=C\left|\overline{v_{h}^{m}}\right|_{1, K}^{2} .
\end{aligned}
$$

Then, with the trace theorem and definition of $\widetilde{v_{h}^{m}}$, we have

$$
\begin{gathered}
\int_{\Omega^{c}}\left(\left.\left\{v_{h}^{m}(x, y)\right\}\right|_{\Omega^{c}}\right)^{2} d s \leq \sum_{K \in \mathcal{T}_{h}} \int_{\partial K}\left(v_{h}^{m}\right)^{2} d s=\sum_{K \in \mathcal{T}_{h}} \int_{\partial K}\left(\widetilde{v_{h}^{m}}+\overline{v_{h}^{m}}\right)^{2} d s \\
\leq C\left(\left\|\widetilde{v_{h}^{m}}\right\|_{1, \Omega^{m}}^{2}+\sum_{K \in \mathcal{T}_{h}} \int_{\partial K}\left|\overline{v_{h}^{m}}\right|^{2} d s\right) \leq C\left(\left|\widetilde{v_{h}^{m}}\right|_{1, \Omega^{m}}^{2}+\sum_{K \in \mathcal{T}_{h}}\left|\overline{v_{h}^{m}}\right|_{1, K}^{2}\right) \\
\leq C \sum_{K \in \mathcal{T}_{h}}\left(\left|\widetilde{v_{h}^{m}}\right|_{1, K}^{2}+\left|\overline{v_{h}^{m}}\right|_{1, K}^{2}\right) \leq C \sum_{K \in \mathcal{T}_{h}}\left|v_{h}^{m}\right|_{1, K}^{2} .
\end{gathered}
$$

Therefore, for all $\mathbf{u}_{h}=\left(u_{h}^{m}, u_{h}^{c}\right) \in V_{h} \times W_{h}$, it follows from (2.11) that

$$
\begin{aligned}
\left|a_{h}\left(\mathbf{u}_{h}, \mathbf{v}_{h}\right)\right|= & \mid \sum_{K \in \mathcal{T}_{h}} \int_{K} \mathbb{K} \nabla u_{h}^{m} \nabla v_{h}^{m} d x d y+\int_{\Omega^{c}} D \frac{\partial u_{h}^{c}}{\partial \tau} \frac{\partial v_{h}^{c}}{\partial \tau} d s \\
& +\alpha_{e x} \int_{\Omega^{c}}\left(\left\{u_{h}^{m}\right\} \mid \Omega_{\Omega^{c}}-u_{h}^{c}\right)\left(\left.\left\{v_{h}^{m}\right\}\right|_{\Omega^{c}}-v_{h}^{c}\right) d s \mid \\
\leq & C\left(\sum_{K \in \mathcal{T}_{h}}\left|u_{h}^{m}\right|_{1, K}\left|v_{h}^{m}\right|_{1, K}+\left\|u_{h}^{c}\right\|_{1, \Omega^{c}}\left\|v_{h}^{c}\right\|_{1, \Omega^{c}}\right. \\
& \left.+\sum_{K \in \mathcal{T}_{h}}\left|u_{h}^{m}\right|_{1, K}\left\|v_{h}^{c}\right\|_{1, \Omega^{c}}+\left\|u_{h}^{c}\right\|_{1, \Omega^{c}} \sum_{K \in \mathcal{T}_{h}}\left|v_{h}^{m}\right|_{1, K}\right) \\
\leq & C\left(\sum_{K \in \mathcal{T}_{h}}\left|u_{h}^{m}\right|_{1, K}+\left\|u_{h}^{c}\right\|_{1, \Omega^{c}}\right)\left(\sum_{K \in \mathcal{T}_{h}}\left|v_{h}^{m}\right|_{1, K}+\left\|v_{h}^{c}\right\|_{1, \Omega^{c}}\right) \\
= & C\left\|\mathbf{u}_{h}\right\|_{1, h}\left\|\mathbf{v}_{h}\right\|_{1, h} .
\end{aligned}
$$

By the Lax-Milgram theorem, we know (2.5) exists a unique solution $\mathbf{h}_{h}$. 


\section{$3 \quad$ Error estimates}

In this section, we shall present the optimal error estimates in $L^{2}$ norm and $H^{1}$ norm for the approximation to the CCPF model.

Firstly, we introduce some interpolation operators of quasi-Wilson nonconforming element on the domain $\Omega^{m}$ as follows. On the reference element, denote the bilinear interpolation operator $\hat{\Pi}_{K}^{m}: H^{2}(\hat{K}) \rightarrow P_{2}(\hat{K})$ by

$$
\hat{\Pi}_{K}^{m} \hat{v}^{m}=\sum_{i=1}^{4} \hat{N}_{i}(\hat{x}, \hat{y}) \hat{v}_{i}^{m} .
$$

Then, it follows from Lemma 3 in [1] and Theorem 2.2 in [8] that the given interpolation operator (3.1) satisfies the following estimate without the regularity assumption (2.2)

$$
\left\|\hat{D}^{\alpha}\left(\hat{v}^{m}-\hat{\Pi}_{K}^{m} \hat{v}^{m}\right)\right\|_{0, \hat{K}} \leq C(\hat{K})\left|\hat{D}^{\alpha} \hat{v}^{m}\right|_{1, \hat{K}},
$$

where $\alpha$ is a multi-index and $|\alpha|=1$.

And on the physical element, the interpolation operators are defined as

$$
\Pi_{K}^{m}: H^{2}(K) \rightarrow P_{2}(K) \quad \text { with } \quad \Pi_{K}^{m} v^{m}=\left(\hat{\Pi}_{K}^{m} \hat{v}^{m}\right) \circ F_{K}^{-1} .
$$

Then define

$$
\Pi^{m}: H^{2}\left(\Omega^{m}\right) \rightarrow V_{h} \quad \text { with }\left.\quad \Pi^{m}\right|_{K}=\Pi_{K}^{m} .
$$

Due to the definition (2.7) we have that

$$
\begin{aligned}
\left\|v^{m}-\Pi^{m} v^{m}\right\|_{1, h} & =\left(\sum_{K \in \mathcal{T}_{h}}\left|v^{m}-\Pi_{K}^{m} v^{m}\right|_{1, K}^{2}\right)^{1 / 2} \\
& =\left(\sum_{K \in \mathcal{T}_{h}} \sum_{|\alpha|=1}\left\|D^{\alpha}\left(v^{m}-\Pi_{K}^{m} v^{m}\right)\right\|_{0, K}^{2}\right)^{1 / 2},
\end{aligned}
$$

from the property $(3.2)$,

$$
\begin{aligned}
\| v^{m}- & \Pi^{m} v^{m} \|_{1, h} \leq C\left(\sum_{K \in \mathcal{T}_{h}} \sum_{|\alpha|=1} \mathbf{h}_{K}^{-2 \alpha}\left\|\hat{D}^{\alpha}\left(\hat{v}^{m}-\hat{\Pi}_{K}^{m} \hat{v}^{m}\right)\right\|_{0, \hat{K}}^{2}\left(h_{x, K} h_{y, K}\right)\right)^{1 / 2} \\
& \leq C\left(\sum_{K \in \mathcal{T}_{h}} \sum_{|\alpha|=1} \mathbf{h}_{K}^{-2 \alpha}\left|\hat{D}^{\alpha} \hat{v}^{m}\right|_{1, \hat{K}^{2}}^{2}\left(h_{x, K} h_{y, K}\right)\right)^{1 / 2} \\
& \leq C\left(\sum_{K \in \mathcal{T}_{h}} \sum_{|\alpha|=1} \mathbf{h}_{K}^{-2 \alpha} \sum_{|\beta|=1}\left\|\hat{D}^{\alpha+\beta} \hat{v}^{m}\right\|_{0, \hat{K}}^{2}\left(h_{x, K} h_{y, K}\right)\right)^{1 / 2} \\
& \leq C\left(\sum_{K \in \mathcal{T}_{h}} \sum_{|\alpha|=1} \sum_{|\beta|=1} \mathbf{h}_{K}^{2 \beta}\left\|D^{\alpha+\beta} v^{m}\right\|_{0, K}^{2}\right)^{1 / 2} \\
\leq & C \sum_{K \in \mathcal{T}_{h}} \sum_{|\alpha|=1} \mathbf{h}_{K}^{\alpha}\left|D^{\alpha} v^{m}\right|_{1, K}
\end{aligned}
$$


where $\mathbf{h}_{K}^{\alpha}=h_{x, K}^{\alpha_{1}} h_{y, K}^{\alpha_{2}}$ with $\alpha=\left(\alpha_{1}, \alpha_{2}\right)$.

For the pipe domain $\Omega^{c}$, the piecewise linear interpolation operator $\Pi^{c}$ : $H^{2}\left(\Omega^{c}\right) \rightarrow W_{h}$ has the property

$$
\left\|v^{c}-\Pi{ }^{c} v^{c}\right\|_{1, \Omega^{c}} \leq C h_{x, K}\left\|v^{c}\right\|_{2, \Omega^{c}},
$$

where $h_{x, K}$ is maximum mesh step of the subdivision of pipe region obtained by $\mathcal{T}_{h}$ restriction on $\Omega^{c}$.

Thus, a valuable lemma is given as basis of error derivation.

Lemma 2. For any $w_{h}^{m} \in V_{h}, w_{h}^{m}=\overline{w_{h}^{m}}+\widetilde{w_{h}^{m}}$, where $\overline{w_{h}^{m}}$ is the nonconforming part and $\widetilde{w_{h}^{m}}$ is the conforming part, there exists a positive constant $C$ such that

$$
\left\|\nabla \overline{w_{h}^{m}}\right\|_{0, K} \leq C\left|w_{h}^{m}\right|_{1, K}, \quad\left\|\overline{w_{h}^{m}}\right\|_{0, K} \leq C h_{K}\left|w_{h}^{m}\right|_{1, K} .
$$

Proof. From the definition (2.7) and the property of affine transformation

$$
\begin{aligned}
\left\|\nabla \overline{w_{h}^{m}}\right\|_{0, K}^{2}=\left|\overline{w_{h}^{m}}\right|_{1, K}^{2} & \leq C h_{x, K} h_{y, K} \sum_{|\beta|=1} \mathbf{h}_{K}^{-2 \beta}\left\|\hat{D}^{\beta} \hat{w}_{h}^{m}\right\|_{0, \hat{K}}^{2} \\
& \leq C h_{x, K} h_{y, K} \sum_{|\beta|=1}^{\mid} \mathbf{h}_{K}^{-2 \beta}\left\|\hat{D}^{\beta} \hat{w}_{h}^{m}\right\|_{0, \hat{K}}^{2} \\
& \leq C \sum_{|\beta|=1}\left\|D^{\beta} w_{h}^{m}\right\|_{0, K}^{2} \leq C\left|w_{h}^{m}\right|_{1, K}^{2} .
\end{aligned}
$$

Using (2.9) and (2.10), and with the same techniques as (3.5), we have

$$
\begin{aligned}
\left\|\overline{w_{h}^{m}}\right\|_{0, K}^{2} & \leq C h_{x, K} h_{y, K}\left\|\overline{\hat{w}_{h}^{m}}\right\|_{0, \hat{K}}^{2} \leq C h_{x, K} h_{y, K}\left|\overline{\hat{w}_{h}^{m}}\right|_{1, \hat{K}}^{2} \\
& \leq C h_{x, K} h_{y, K}\left|\hat{w}_{h}^{m}\right|_{1, \hat{K}}^{2} \leq C h_{K}^{2}\left|w_{h}^{m}\right|_{1, K}^{2} .
\end{aligned}
$$

Lemma 3. Assume $\mathbf{h}=\left(h^{m}, h^{c}\right)$ and $\mathbf{h}_{h}=\left(h_{h}^{m}, h_{h}^{c}\right)$ are defined by (2.1) and (2.5), respectively. Then, we have the following error estimate

$$
\begin{aligned}
\left\|\mathbf{h}-\mathbf{h}_{h}\right\|_{1, h} \leq & C\left(\operatorname{vinf}_{\mathbf{v}_{h}=\left(v_{h}^{m}, v_{h}^{c}\right) \in V_{h} \times W_{h}}\left\|\mathbf{h}-\mathbf{v}_{h}\right\|_{1, h}\right. \\
& \left.+\operatorname{w}_{\mathbf{w}_{h}=\left(w_{h}^{m}, w_{h}^{c}\right) \in V_{h} \times W_{h} \backslash\{\mathbf{0}\}} \frac{\left|a_{h}\left(\mathbf{h}, \mathbf{w}_{h}\right)-\left(\mathbf{f}, \mathbf{w}_{h}\right)\right|}{\left\|\mathbf{w}_{h}\right\|_{1, h}}\right) .
\end{aligned}
$$

Proof. Since

$$
\left\|\mathbf{h}-\mathbf{h}_{h}\right\|_{1, h} \leq\left\|\mathbf{h}-\mathbf{v}_{h}\right\|_{1, h}+\left\|\mathbf{v}_{h}-\mathbf{h}_{h}\right\|_{1, h}, \quad \forall \mathbf{v}_{h}=\left(v_{h}^{m}, v_{h}^{c}\right) \in V_{h} .
$$

Then, we need to estimate the right hand side of (3.7). In view of the continuity, coercivity of $a_{h}(\cdot, \cdot)$ in Theorem 1 and definition of $(2.5)$, we have

$$
\begin{aligned}
C_{1}\left\|\mathbf{v}_{h}-\mathbf{h}_{h}\right\|_{1, h}^{2} & \leq a_{h}\left(\mathbf{v}_{h}-\mathbf{h}_{h}, \mathbf{v}_{h}-\mathbf{h}_{h}\right) \\
& \leq a_{h}\left(\mathbf{v}_{h}-\mathbf{h}, \mathbf{v}_{h}-\mathbf{h}_{h}\right)+a_{h}\left(\mathbf{h}-\mathbf{h}_{h}, \mathbf{v}_{h}-\mathbf{h}_{h}\right) \\
& \leq C_{2}\left\|\mathbf{h}-\mathbf{v}_{h}\right\|_{1, h}\left\|\mathbf{v}_{h}-\mathbf{h}_{h}\right\|_{1, h}+a_{h}\left(\mathbf{h}, \mathbf{v}_{h}-\mathbf{h}_{h}\right)-\left(\mathbf{f}, \mathbf{v}_{h}-\mathbf{h}_{h}\right) .
\end{aligned}
$$


If $\mathbf{v}_{h} \neq \mathbf{h}_{h}$, then

$$
\begin{gathered}
\left\|\mathbf{v}_{h}-\mathbf{h}_{h}\right\|_{1, h} \leq C\left(\left\|\mathbf{h}-\mathbf{v}_{h}\right\|_{1, h}+\frac{\left|a_{h}\left(\mathbf{h}, \mathbf{v}_{h}-\mathbf{h}_{h}\right)-\left(\mathbf{f}, \mathbf{v}_{h}-\mathbf{h}_{h}\right)\right|}{\left\|\mathbf{v}_{h}-\mathbf{h}_{h}\right\|_{1, h}}\right) \\
\leq C\left(\left\|\mathbf{h}-\mathbf{v}_{h}\right\|_{1, h}+\sup _{\mathbf{w}_{h} \in\left(V_{h} \times W_{h}\right) \backslash\{\mathbf{0}\}} \frac{\left|a_{h}\left(\mathbf{h}, \mathbf{w}_{h}\right)-\left(\mathbf{f}, \mathbf{w}_{h}\right)\right|}{\left\|\mathbf{w}_{h}\right\|_{1, h}}\right) .
\end{gathered}
$$

Furthermore, because $\mathbf{w}_{h}=\left(w_{h}^{m}, w_{h}^{c}\right)=\left(\overline{w_{h}^{m}}+\widetilde{w_{h}^{m}}, w_{h}^{c}\right)$ and $\widetilde{w_{h}^{m}}, w_{h}^{c}$ are conforming parts, then

$$
\begin{aligned}
& a_{h}\left(\mathbf{h}, \mathbf{w}_{h}\right)-\left(\mathbf{f}, \mathbf{w}_{h}\right)=\sum_{K \in \mathcal{T}_{h}} \int_{K} \mathbb{K} \nabla h^{m} \nabla \overline{w_{h}^{m}} d x d y \\
& \quad+\left.\alpha_{e x} \int_{\Omega^{c}}\left(\left.\left\{h^{m}\right\}\right|_{\Omega^{c}}-h^{c}\right)\left\{\overline{w_{h}^{m}}\right\}\right|_{\Omega^{c}} d s-\int_{K} f^{m} \overline{w_{h}^{m}} d x d y .
\end{aligned}
$$

In conclusion, the error estimate (3.6) is obtained by combining (3.8), (3.9) and (3.7).

Denote the whole domain $\Omega^{m} \cup \Omega^{c}$ by $\Omega$ and $\|\mathbf{v}\|_{0, \Omega}=\left\|v^{m}\right\|_{0, \Omega^{m}}+\left\|v^{c}\right\|_{0, \Omega^{c}}$. According to Cao et al. [5], in the case of homogeneous isotropic matrix, one can easily check via separation of variable that we have higher order piecewise regularity in the sense that $\left.h^{m}\right|_{\Omega^{m} \cap\{y>k x+b\}} \in H^{2}\left(\Omega^{m} \cap\{y>k x+b\}\right)$ and $\left.h^{m}\right|_{\Omega^{m} \cap\{y<k x+b\}} \in H^{2}\left(\Omega^{m} \cap\{y<k x+b\}\right)$. Then, we derive the following convergence theorem.

Theorem 2. Assume $\mathbf{h}=\left(h^{m}, h^{c}\right)$ and $\mathbf{h}_{h}=\left(h_{h}^{m}, h_{h}^{c}\right)$ defined by (2.1) and (2.5), respectively. We get

$$
\begin{aligned}
\left\|\mathbf{h}-\mathbf{h}_{h}\right\|_{1, h} \leq & C\left(\sum_{K \in \mathcal{T}_{h}} \sum_{|\alpha|=1} \mathbf{h}_{K}^{\alpha}\left|D^{\alpha} h^{m}\right|_{1, K}\right. \\
& \left.+h\left(\left\|h^{c}\right\|_{2, \Omega^{c}}+\left\|f^{c}\right\|_{0, \Omega^{c}}+\left\|f^{m}\right\|_{0, \Omega^{m}}\right)\right), \\
\left\|\mathbf{h}-\mathbf{h}_{h}\right\|_{0, \Omega} \leq & C h\left(\sum_{K \in \mathcal{T}_{h}|\alpha|=1} \sum_{K} \mathbf{h}_{K}^{\alpha}\left|D^{\alpha} h^{m}\right|_{1, K}\right. \\
& \left.+h\left(\left\|h^{c}\right\|_{2, \Omega^{c}}+\left\|f^{c}\right\|_{0, \Omega^{c}}+\left\|f^{m}\right\|_{0, \Omega^{m}}\right)\right) .
\end{aligned}
$$

Proof. By Lemma 3, we need to estimate the right hand of (3.6). The first term on the right hand side, which is interpolation error, can be obtained from the interpolation properties (3.3) and (3.4),

$$
\begin{aligned}
\inf _{\mathbf{v}_{h} \in V_{h} \times W_{h}}\left\|\mathbf{h}-\mathbf{v}_{h}\right\|_{1, h} & \leq\left\|\mathbf{h}-\mathbf{\Pi} \mathbf{v}_{h}\right\|_{1, h} \\
& \leq\left\|h^{m}-\Pi^{m} h^{m}\right\|_{1, h}+\left\|h^{c}-\Pi^{c} h^{c}\right\|_{1, \Omega^{c}} \\
& \leq C\left(\sum_{K \in \mathcal{T}_{h}} \sum_{|\alpha|=1} \mathbf{h}_{K}^{\alpha}\left|D^{\alpha} h^{m}\right|_{1, K}+h\left\|h^{c}\right\|_{2, \Omega^{c}}\right),
\end{aligned}
$$


where $\boldsymbol{\Pi}=\left(\Pi^{m}, \Pi^{c}\right)$ and $C$ is independent of $h_{K} / \rho_{K}$.

From the definition (2.8) and affine transformation (2.3)-(2.4), we have

$$
\int_{K} \nabla \overline{v_{h}^{m}} d x d y=\int_{\hat{K}} \nabla \overline{\hat{v}_{h}^{m}}\left|J_{K}\right| d \hat{x} d \hat{y}=0 .
$$

Hence, for the second term on the right hand side, we can obtain based on Lemma 3 and Hölder inequality that

$$
\begin{aligned}
\left|a_{h}\left(\mathbf{h}, \mathbf{w}_{h}\right)-\left(\mathbf{f}, \mathbf{w}_{h}\right)\right|=\mid \sum_{K \in \mathcal{T}_{h}} \int_{K} \mathbb{K} \nabla h^{m} \nabla \overline{w_{h}^{m}} d x d y \\
\quad+\left.\alpha_{e x} \int_{\Omega^{c}}\left(\left.h^{m}\right|_{\Omega^{c}}-h^{c}\right)\left\{\overline{w_{h}^{m}}\right\}\right|_{\Omega^{c}} d s-\int_{K} f^{m} \overline{w_{h}^{m}} d x d y \mid \\
\leq C\left(\left|\sum_{K \in \mathcal{T}_{h}} \int_{K} \mathbb{K}\left(\nabla h^{m}-M\left(\nabla h^{m}\right)\right) \nabla \overline{w_{h}^{m}} d x d y\right|\right. \\
\left.\quad+\left|\int_{\Omega^{c}}\left(h^{m}{\mid \Omega^{c}}-h^{c}\right)\left\{\overline{w_{h}^{m}}\right\}\right| \Omega^{c} d s|+| \int_{K} f^{m} \overline{w_{h}^{m}} d x d y \mid\right),
\end{aligned}
$$

where $M\left(\nabla \varphi^{m}\right)=\frac{1}{\operatorname{meas}(K)} \int_{K} \nabla \varphi^{m} d x d y$.

Then, from the trace theorem and (2.11),

$$
\begin{aligned}
\left|a_{h}\left(\mathbf{h}, \mathbf{w}_{h}\right)-\left(\mathbf{f}, \mathbf{w}_{h}\right)\right| \leq & C\left(\sum_{K \in \mathcal{T}_{h}} \sum_{|\alpha|=1} \mathbf{h}_{K}^{\alpha}\left|D^{\alpha} h^{m}\right|_{1, K}+h\left(\left\|h^{c}\right\|_{2, \Omega^{c}}\right.\right. \\
& \left.\left.+\left\|f^{c}\right\|_{0, \Omega^{c}}+\left\|f^{m}\right\|_{0, \Omega^{m}}\right)\right)\left|w_{h}^{m}\right|_{1, K} \\
\leq & C\left(\sum_{K \in \mathcal{T}_{h}} \sum_{|\alpha|=1} \mathbf{h}_{K}^{\alpha}\left|D^{\alpha} h^{m}\right|_{1, K}+h\left(\left\|h^{c}\right\|_{2, \Omega^{c}}\right.\right. \\
& \left.\left.+\left\|f^{c}\right\|_{0, \Omega^{c}}+\left\|f^{m}\right\|_{0, \Omega^{m}}\right)\right)\left|\mathbf{w}_{h}\right|_{1, K} .
\end{aligned}
$$

Therefore, (3.10) is established from (3.12) and (3.14).

In order to derive the errors in $L^{2}$ norm, the adjoint problem is considered for a duality argument. Let $\mathbf{u}=\left(u^{m}, u^{c}\right) \in L^{2}\left(\Omega^{m}\right) \times L^{2}\left(\Omega^{c}\right)$ and $\boldsymbol{\Psi}=$ $\left(\varphi^{m}, \varphi^{c}\right) \in H_{0}^{1}\left(\Omega^{m}\right) \times H_{0}^{1}\left(\Omega^{c}\right)$ satisfy

$$
\begin{cases}-\nabla \cdot\left(\mathbb{K} \nabla \varphi^{m}\right)=-\alpha_{e x}\left(\varphi^{m}-\varphi^{c}\right) \delta_{\Omega^{c}}+u^{m}, & \text { in } \Omega^{m} \\ -\frac{\partial}{\partial \tau}\left(D \frac{\partial \varphi^{c}}{\partial \tau}\right)=\alpha_{e x}\left(\left.\varphi^{m}\right|_{\Omega^{c}}-\varphi^{c}\right)+u^{c}, & \text { in } \quad \Omega^{c}\end{cases}
$$

with the regularity [5]

$$
\begin{aligned}
& \left\|\varphi^{m}\right\|_{2, \Omega^{m} \cap\{y<k x+b\}}+\left\|\varphi^{m}\right\|_{2, \Omega^{m} \cap\{y>k x+b\}}+\left\|h^{c}\right\|_{2, \Omega^{c}} \\
& \leq C\left(\left\|u^{m}\right\|_{0, \Omega^{m}}+\left\|u^{c}\right\|_{0, \Omega^{c}}\right) .
\end{aligned}
$$

Since

$$
\left\|\mathbf{h}-\mathbf{h}_{h}\right\|_{0, \Omega}=\sup _{\mathbf{u} \in L^{2}(\Omega)} \frac{\left(\mathbf{u}, \mathbf{h}-\mathbf{h}_{h}\right)}{\|\mathbf{u}\|_{0, \Omega}}
$$


we estimate $\left(\mathbf{u}, \mathbf{h}-\mathbf{h}_{h}\right)$.

By Green's formula and noticing that $h^{m}, \widetilde{h_{h}^{m}} \in C^{0}\left(\Omega^{m}\right)$,

$$
\begin{aligned}
\left(\mathbf{u}, \mathbf{h}-\mathbf{h}_{h}\right)=-\sum_{K \in \mathcal{T}_{h}} \int_{K} \nabla \cdot\left(\mathbb{K} \nabla \varphi^{m}\right)\left(h^{m}-h_{h}^{m}\right) d x d y \\
\quad+\int_{\Omega^{c}} D \frac{\partial \varphi^{c}}{\partial \tau} \frac{\partial\left(h^{c}-h_{h}^{c}\right)}{\partial \tau} d s+\alpha_{e x} \int_{\Omega^{c}}\left(\left.\varphi^{m}\right|_{\Omega^{c}}-\varphi^{c}\right)\left(\left.h^{m}\right|_{\Omega^{c}}-\left.\left\{h_{h}^{m}\right\}\right|_{\Omega^{c}}\right) d s \\
\quad-\alpha_{e x} \int_{\Omega^{c}}\left(\left.\varphi^{m}\right|_{\Omega^{c}}-\varphi^{c}\right)\left(h^{c}-h_{h}^{c}\right) d s \\
=\sum_{K \in \mathcal{T}_{h}} \int_{K} \mathbb{K} \nabla \varphi^{m} \nabla\left(h^{m}-h_{h}^{m}\right) d x d y+\int_{\Omega^{c}} D \frac{\partial \varphi^{c}}{\partial \tau} \frac{\partial\left(h^{c}-h_{h}^{c}\right)}{\partial \tau} d s \\
\quad+\alpha_{e x} \int_{\Omega^{c}}\left(\left.\varphi^{m}\right|_{\Omega^{c}}-\varphi^{c}\right)\left(\left.h^{m}\right|_{\Omega^{c}}-\left.\left\{h_{h}^{m}\right\}\right|_{\Omega^{c}}\right) d s \\
\quad-\alpha_{e x} \int_{\Omega^{c}}\left(\left.\varphi^{m}\right|_{\Omega^{c}}-\varphi^{c}\right)\left(h^{c}-h_{h}^{c}\right) d s-\sum_{K \in \mathcal{T}_{h}} \int_{\partial K} \mathbb{K} \frac{\partial \varphi^{m}}{\partial n}\left(h^{m}-h_{h}^{m}\right) d s \\
=a_{h}\left(\boldsymbol{\Psi}, \mathbf{h}-\mathbf{h}_{h}\right)-\sum_{K \in \mathcal{T}_{h}} \int_{\partial K} \mathbb{K} \frac{\partial \varphi^{m}}{\partial n}\left(h^{m}-h_{h}^{m}\right) d s .
\end{aligned}
$$

Then, we need to estimate the following two terms.

Due to the properties (3.3), (3.4), the continuity of interpolation operators $\Pi^{m}$ and $\Pi^{c}$ and the elliptic regularity of this problem (3.15), we know

$$
\begin{aligned}
& \left|a_{h}\left(\boldsymbol{\Psi}, \mathbf{h}-\mathbf{h}_{h}\right)\right| \leq C\|\boldsymbol{\Psi}-\boldsymbol{\Pi} \boldsymbol{\Psi}\|_{1, h}\left\|\mathbf{h}-\mathbf{h}_{h}\right\|_{1, h} \\
& \quad \leq C h\left(\left\|\varphi^{m}\right\|_{2, \Omega^{m} \cap\{y<k x+b\}}+\left\|\varphi^{m}\right\|_{2, \Omega^{m} \cap\{y>k x+b\}}+\left\|\varphi^{c}\right\|_{2, \Omega^{c}}\right)\left\|\mathbf{h}-\mathbf{h}_{h}\right\|_{1, h} \\
& \quad \leq C h\|\mathbf{u}\|_{0, \Omega}\left(\sum_{K \in \mathcal{T}_{h}} \sum_{|\alpha|=1} \mathbf{h}_{K}^{\alpha}\left|D^{\alpha} h^{m}\right|_{1, K}+h\left\|h^{c}\right\|_{2, \Omega^{c}}\right) .
\end{aligned}
$$

Assume $\phi_{h}^{m}=h_{h}^{m}-\Pi^{m} h^{m}$, then $\widetilde{\phi_{h}^{m}}=\widetilde{h_{h}^{m}}-\Pi^{m} h^{m}$ and $\overline{\phi_{h}^{m}}=\overline{h_{h}^{m}}$. We obtain from Lemma 2,

$$
\begin{aligned}
& \left|\sum_{K \in \mathcal{T}_{h}} \int_{\partial K} \mathbb{K} \frac{\partial \varphi^{m}}{\partial n}\left(h^{m}-h_{h}^{m}\right) d s\right|=\sum_{K \in \mathcal{T}_{h}} \mid \int_{K} \nabla \cdot\left(\mathbb{K} \nabla \varphi^{m}\right) \overline{h_{h}^{m}} d x d y \\
& \quad-\int_{K} \mathbb{K} \nabla \varphi^{m} \nabla \overline{h_{h}^{m}} d x d y \mid \leq C \sum_{K \in \mathcal{T}_{h}}\left(\left\|\varphi^{m}\right\|_{2, \Omega^{m} \cap\{y<k x+b\}}\right. \\
& \left.\quad+\left\|\varphi^{m}\right\|_{2, \Omega^{m} \cap\{y>k x+b\}}\right)\left\|\overline{h_{h}^{m}}\right\|_{0, K}+C \sum_{K \in \mathcal{T}_{h}}\left|\int_{K}\left(\nabla \varphi^{m}-M\left(\nabla \varphi^{m}\right)\right) \nabla \overline{h_{h}^{m}} d x d y\right|,
\end{aligned}
$$

then,

$$
\begin{aligned}
& \left|\sum_{K \in \mathcal{T}_{h}} \int_{\partial K} \mathbb{K} \frac{\partial \varphi^{m}}{\partial n}\left(h^{m}-h_{h}^{m}\right) d s\right| \\
\leq & C h\left(\left\|\varphi^{m}\right\|_{2, \Omega^{m} \cap\{y<k x+b\}}+\left\|\varphi^{m}\right\|_{2, \Omega^{m} \cap\{y>k x+b\}}\right)\left\|\phi_{h}^{m}\right\|_{1, h} \\
\leq & C h\left(\left\|\varphi^{m}\right\|_{2, \Omega^{m} \cap\{y<k x+b\}}+\left\|\varphi^{m}\right\|_{2, \Omega^{m} \cap\{y>k x+b\}}\right)
\end{aligned}
$$




$$
\begin{aligned}
& \left(\left\|h^{m}-h_{h}^{m}\right\|_{1, h}+\left\|h^{m}-\Pi^{m} h_{h}^{m}\right\|_{1, h}\right) \\
\leq & C h\left\|u^{m}\right\|_{0, \Omega^{m}}\left(\sum_{K \in \mathcal{T}_{h}} \sum_{|\alpha|=1} \mathbf{h}_{K}^{\alpha}\left|D^{\alpha} h^{m}\right|_{1, K}+h\left\|h^{c}\right\|_{2, \Omega^{c}}\right) \\
\leq & C h\|\mathbf{u}\|_{0, \Omega}\left(\sum_{K \in \mathcal{T}_{h}} \sum_{|\alpha|=1} \mathbf{h}_{K}^{\alpha}\left|D^{\alpha} h^{m}\right|_{1, K}+h\left\|h^{c}\right\|_{2, \Omega^{c}}\right) .
\end{aligned}
$$

Consequently, (3.11) follows from (3.16)-(3.19).

\section{Numerical examples}

In this section, we give some numerical examples using the quasi-Wilson element for Darcy model in porous media and conforming finite element for pipe-flow model in the pipe cube region. In order to verify the rates of convergence, we carry out two examples using two different quadrilateral meshes in two-dimensional domain, respectively. For the sake of simplicity, the domain $\Omega^{m}$ is defined as $(0,1) \times(-0.5,0.5)$ and the pipe $\Omega^{c}$ as $\{y=x / 4, x \in[0,1]\}$. Here, we take $\mathbb{K}=\mathbb{I}$ and $D=1$.

We define two different anisotropic meshes with $m \times n$ quadrilateral elements for porous media $\Omega^{m}$. On mesh 1 , based on two parts of $\Omega^{m}$ dividing by $\Omega^{c}$, we divide each edge along $y$-direction into equal segments. On mesh 2 , when $x=0$, on $y$-direction, divide $[-0.5,0.5]$ into $m$ segments with $m+1$ points $\left\{y_{i}\right\}$, where

$$
\begin{aligned}
& y_{i}=-0.5+0.5 \sin (i \pi / m), \quad i=0,1, \cdots, m / 2, \\
& y_{i}=0.5-0.5 \cos (i \pi / m-\pi / 2), \quad i=m / 2+1, \cdots, m .
\end{aligned}
$$

When $x=1$, on $y$-direction, divide $[-0.5,0.5]$ into $m$ segments with $m+1$ points $\left\{y_{j}\right\}$, too, and

$$
\begin{aligned}
& y_{j}=-0.5+(0.5+1 / 4) \sin (j \pi / m), \quad j=0,1, \cdots, m / 2, \\
& y_{j}=0.5-0.25 \cos (j \pi / m-\pi / 2), \quad j=m / 2+1, \cdots, m .
\end{aligned}
$$

For all cases, along $x$-direction, we divide $[0,1]$ into $n$ equal cells with $n+1$ points, see Figure 3.

Example 1. The analytic solution is chosen as

$$
\left\{\begin{array}{lll}
h^{c}=\left(\frac{3 \sqrt{17}}{2}+1\right) \sin (4 \pi x), & \text { in } \quad \Omega^{c}, \\
h^{m}=e^{3 y-\frac{3 x}{4}} \sin (4 \pi x), & \text { in } \quad(0,1) \times(-1 / 2,0] \in \Omega^{m}, \\
h^{m}=e^{-3 y+\frac{3 x}{4} \sin (4 \pi x),} & \text { in } \quad(0,1) \times[0,1 / 2) \in \Omega^{m},
\end{array}\right.
$$

with $\alpha_{e x}=4+\sin (4 \pi x)$.

Example 2. The analytic solution is chosen as

$$
\begin{cases}h^{c}=(\sqrt{17} / 4+1-0.25 x) \sin (2 \pi x), & \text { in } \Omega^{c}, \\ h^{m}=(1-0.25 x) \sin (2 \pi x), & \text { in }(0,1) \times(-1 / 2,0] \in \Omega^{m}, \\ h^{m}=(1-y) \sin (2 \pi x), & \text { in } \quad(0,1) \times[0,1 / 2) \in \Omega^{m},\end{cases}
$$



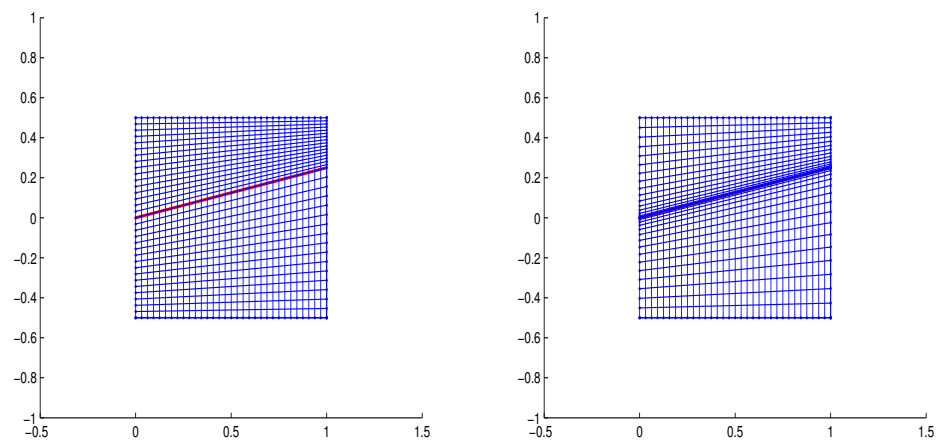

Figure 3. The anisotropic meshes with $m=n=32$. Right: anisotropic mesh 1; left: anisotropic mesh 2.

with $\alpha_{e x}=2+\sin (2 \pi x)$.

Let $\left(E_{1}^{m}, E_{1}^{c}\right)$ and $\left(E_{2}^{m}, E_{2}^{c}\right)$ denote the relative errors by applying the quasiWilson nonconforming element to solve Darcy equation with the 1-D conforming element to solve pipe-flow equation on anisotropic mesh 1 and mesh 2 , respectively. In reality different mesh sizes with different values of $n=m$ are used. The errors are listed in the following Figure 4 - Figure 7 and Table $1-$ Table 2.
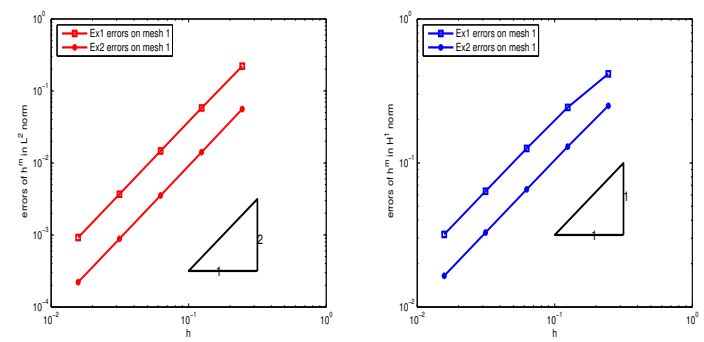

Figure 4. Errors of $h^{m}$ on mesh 1 in $L^{2}$ norm and $H^{1}$ norm. Right: errors in $L^{2}$ norm; left: errors in $H^{1}$ norm.

From the above figures and Table 1 - Table 2, we can see the almost 2-nd order convergence rate in $L^{2}$ norm and the 1-st order convergence rate in $H^{1}$ norm for $h^{m}$ and $h^{c}$ by quasi-Wilson element and conforming finite element method, respectively, which are consistent with our theoretical results. Moreover, the optimal error estimates are independent of the value of $\left\{\frac{h_{K}}{\rho_{K}}\right\}$, which means that we can get the same order of errors rates whether the subdivisions satisfy the regularity condition or not.

By comparison anisotropic mesh 1 with anisotropic mesh 2 the computational errors in $L^{2}$ norm on the former mesh are better than on the latter mesh, but the errors in $H^{1}$ norm on the former mesh are worse than on the latter mesh. Therefore, both anisotropic meshes have their advantages and 

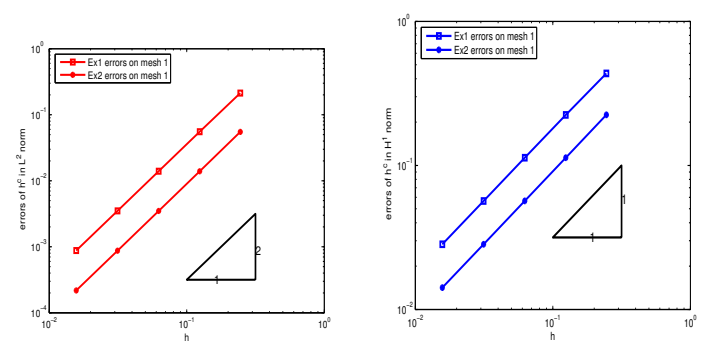

Figure 5. Errors of $h^{c}$ on mesh 1 in $L^{2}$ norm and $H^{1}$ norm. Right: errors in $L^{2}$ norm; left: errors in $H^{1}$ norm.

Table 1. Errors on anisotropic mesh 1.

\begin{tabular}{llllllll}
\hline & $n$ & $h$ & $\left\|E_{1}^{m}\right\|_{0, \Omega^{m}}$ & $\left\|E_{1}^{m}\right\|_{1, h}$ & $\left\|E_{1}^{c}\right\|_{0, \Omega^{c}}$ & $\left\|E_{1}^{c}\right\|_{1, \Omega^{c}}$ & $\max \left\{\frac{h_{K}}{\rho_{K}}\right\}$ \\
\hline Ex1 & 8 & $2.4519 \mathrm{e}-1$ & $2.2059 \mathrm{e}-1$ & $4.1561 \mathrm{e}-1$ & $2.1273 \mathrm{e}-1$ & $4.3524 \mathrm{e}-1$ & 1.0078 \\
& 16 & $1.2428 \mathrm{e}-1$ & $5.8023 \mathrm{e}-2$ & $2.4298 \mathrm{e}-1$ & $5.5331 \mathrm{e}-2$ & $2.2441 \mathrm{e}-1$ & 2.0308 \\
& 32 & $6.2563 \mathrm{e}-2$ & $1.4692 \mathrm{e}-2$ & $1.2613 \mathrm{e}-1$ & $1.3969 \mathrm{e}-2$ & $1.1307 \mathrm{e}-1$ & 4.1780 \\
& 64 & $3.1387 \mathrm{e}-2$ & $3.6849 \mathrm{e}-3$ & $6.3655 \mathrm{e}-2$ & $3.5009 \mathrm{e}-3$ & $5.6645 \mathrm{e}-2$ & 5.1519 \\
& 128 & $1.5720 \mathrm{e}-2$ & $9.2196 \mathrm{e}-4$ & $3.1901 \mathrm{e}-2$ & $8.7577 \mathrm{e}-4$ & $2.8336 \mathrm{e}-2$ & 8.8957 \\
\hline rate & & & 1.9958 & 0.9424 & 2.0007 & 0.9956 & \\
\hline \multirow{2}{*}{ Ex2 } & 8 & $2.4519 \mathrm{e}-1$ & $5.5955 \mathrm{e}-2$ & $2.4942 \mathrm{e}-1$ & $5.5071 \mathrm{e}-2$ & $2.2466 \mathrm{e}-1$ & 1.0078 \\
& 16 & $1.2428 \mathrm{e}-1$ & $1.4136 \mathrm{e}-2$ & $1.2988 \mathrm{e}-1$ & $1.3898 \mathrm{e}-2$ & $1.1319 \mathrm{e}-1$ & 2.0308 \\
& 32 & $6.2563 \mathrm{e}-2$ & $3.5427 \mathrm{e}-3$ & $6.5596 \mathrm{e}-2$ & $3.4827 \mathrm{e}-3$ & $5.6706 \mathrm{e}-2$ & 4.1780 \\
& 64 & $3.1387 \mathrm{e}-2$ & $8.8583 \mathrm{e}-4$ & $3.2880 \mathrm{e}-2$ & $8.7116 \mathrm{e}-4$ & $2.8367 \mathrm{e}-2$ & 5.1519 \\
& 128 & $1.5720 \mathrm{e}-2$ & $2.2106 \mathrm{e}-4$ & $1.6450 \mathrm{e}-2$ & $2.1781 \mathrm{e}-4$ & $1.4185 \mathrm{e}-2$ & 8.8957 \\
\hline rate & & & 2.0141 & 0.9914 & 2.0137 & 1.0056 & \\
\hline
\end{tabular}

Table 2. Errors on anisotropic mesh 2.

\begin{tabular}{llllllll}
\hline & $n$ & $h$ & $\left\|E_{1}^{m}\right\|_{0, \Omega^{m}}$ & $\left\|E_{1}^{m}\right\|_{1, h}$ & $\left\|E_{1}^{c}\right\|_{0, \Omega^{c}}$ & $\left\|E_{1}^{c}\right\|_{1, \Omega^{c}}$ & $\max \left\{\frac{h_{K}}{\rho_{K}}\right\}$ \\
\hline Ex1 & 8 & $3.1305 \mathrm{e}-1$ & $2.1753 \mathrm{e}-1$ & $4.2671 \mathrm{e}-1$ & $2.1273 \mathrm{e}-1$ & $4.3524 \mathrm{e}-1$ & 6.7707 \\
& 16 & $1.5911 \mathrm{e}-1$ & $6.2777 \mathrm{e}-2$ & $2.1887 \mathrm{e}-1$ & $5.5330 \mathrm{e}-2$ & $2.2441 \mathrm{e}-1$ & 13.4113 \\
& 32 & $7.9932 \mathrm{e}-2$ & $1.6334 \mathrm{e}-2$ & $1.1013 \mathrm{e}-1$ & $1.3969 \mathrm{e}-2$ & $1.1307 \mathrm{e}-1$ & 26.7580 \\
& 64 & $4.0088 \mathrm{e}-2$ & $4.1250 \mathrm{e}-3$ & $5.5150 \mathrm{e}-2$ & $3.5009 \mathrm{e}-3$ & $5.6645 \mathrm{e}-2$ & 53.4838 \\
& 128 & $2.0070 \mathrm{e}-2$ & $1.0339 \mathrm{e}-3$ & $2.7586 \mathrm{e}-2$ & $8.7576 \mathrm{e}-4$ & $2.8336 \mathrm{e}-2$ & 106.9495 \\
\hline rate & & & 1.9528 & 0.9976 & 2.0000 & 0.9953 & \\
\hline Ex2 & 8 & $3.1305 \mathrm{e}-1$ & $6.1155 \mathrm{e}-2$ & $2.2364 \mathrm{e}-1$ & $5.5071 \mathrm{e}-2$ & $2.2466 \mathrm{e}-1$ & 6.7707 \\
& 16 & $1.5911 \mathrm{e}-1$ & $1.5947 \mathrm{e}-2$ & $1.1266 \mathrm{e}-1$ & $1.3898 \mathrm{e}-2$ & $1.1319 \mathrm{e}-1$ & 13.4113 \\
& 32 & $7.9932 \mathrm{e}-2$ & $4.0294 \mathrm{e}-3$ & $5.6436 \mathrm{e}-2$ & $3.4827 \mathrm{e}-3$ & $5.6706 \mathrm{e}-2$ & 26.7580 \\
& 64 & $4.0088 \mathrm{e}-2$ & $1.0097 \mathrm{e}-3$ & $2.8231 \mathrm{e}-2$ & $8.7116 \mathrm{e}-4$ & $2.8367 \mathrm{e}-2$ & 53.4838 \\
& 128 & $2.0070 \mathrm{e}-2$ & $2.5222 \mathrm{e}-4$ & $1.4117 \mathrm{e}-2$ & $2.1781 \mathrm{e}-4$ & $1.4185 \mathrm{e}-2$ & 106.9495 \\
\hline rate & & & 1.9994 & 1.0053 & 2.0130 & 1.0052 & \\
\hline
\end{tabular}

disadvantages. 

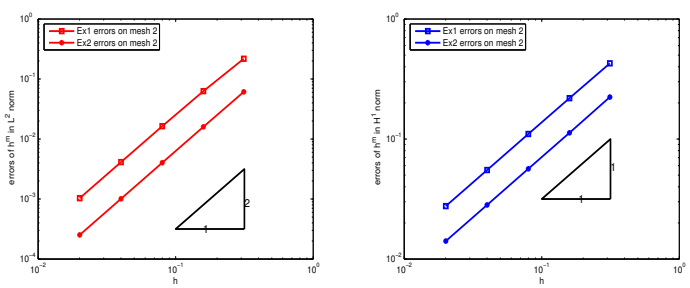

Figure 6. Errors of $h^{m}$ on mesh 2 in $L^{2}$ norm and $H^{1}$ norm. Right: errors in $L^{2}$ norm; left: errors in $H^{1}$ norm.
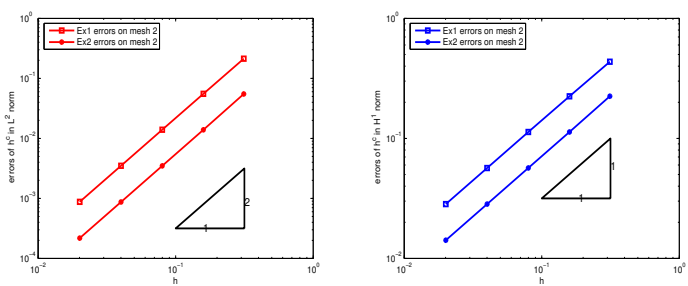

Figure 7. Errors of $h^{c}$ on mesh 2 in $L^{2}$ norm and $H^{1}$ norm. Right: errors in $L^{2}$ norm; left: errors in $H^{1}$ norm.

In order to report the features of the approach introduced in this paper, we present the following Figure 8 - Figure 9 of exact solution $\mathbf{h}=\left(h^{m}, h^{c}\right)$ and numerical solution $\mathbf{h}_{h}=\left(h_{h}^{m}, h_{h}^{c}\right)$ based on the space with the mesh size $h=2^{-7}$, respectively.
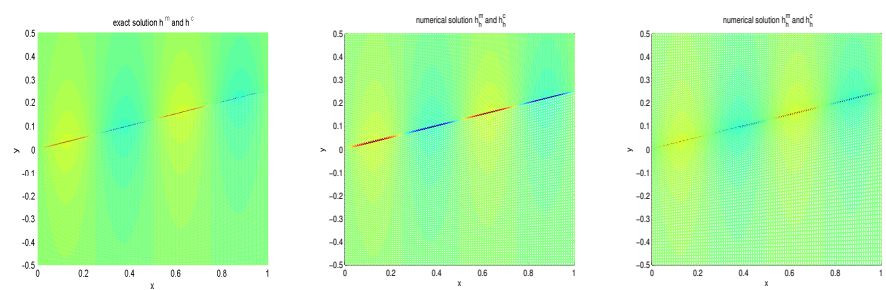

Figure 8. Figures of solution of Darcy model in the porous matrix and pipe-flow model in the conduit region for Example 1. Left: Exact solution; middle: Numerical solution on anisotropic mesh 1; right: Numerical solution on anisotropic mesh 2.

From the figures of exact solution and those of computed solution, it is clear that the approach introduced in this paper is effective to approximate the coupled continuum pipe-flow/Darcy model.

\section{Conclusions}

In this paper, we have presented a numerical method for solving the coupled continuum pipe-flow/Darcy (CCPF) model that is suitable for the study of groundwater flow in Karst aquifers. The two-dimensional and steady-state case 

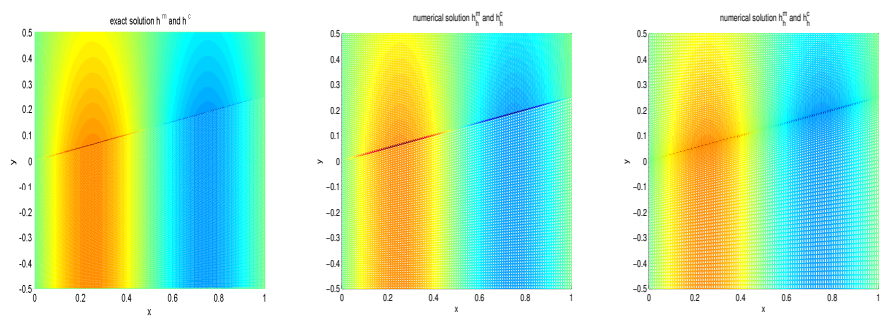

Figure 9. Figures of solution of Darcy model in the porous matrix and pipe-flow model in the conduit region for Example 2. Left: Exact solution; middle: Numerical solution on anisotropic mesh 1; right: Numerical solution on anisotropic mesh 2.

in which the conduit is embedded in the porous media domain at a large angle of inclination is examined, so that one cannot use a traditional regular mesh and finite element method for the Darcy region. Instead, we have employed an anisotropic mesh without a regularity condition and use nonconforming, quasi-Wilson elements to solve the Darcy problem, along with conforming finite element method for the conduit flow problem. On basis of this paper, our future work is to analyze and test the cases of curved pipes and intersect pipes.

\section{Acknowledgements}

The authors thank the anonymous referee for constructive comments and suggestions which led to significant improvement of this paper.

\section{References}

[1] T. Apel and M. Dobrowolski. Anisotropic interpolation with applications to the finite element method. Computing, 47(3):277-293, 1992. http://dx.doi.org/10.1007/BF02320197.

[2] S. Bauer, R. Liedl and M. Sauter. Modelling of karst development considering conduit-matrix exchange flow. In F. Stauffera, W. Kinzelbach, K. Kovar and E. Hoehn(Eds.), Calibration and reliability in groundwater modelling: coping with uncertainty. (Proceedings of the ModelCARE'99 conference held at Z "urich, Switzerland, September 1999), Wallingford, Oxfordshire, UK, 2000. IAHS Publication no. 265.

[3] S. Bauer, R. Liedl and M. Sauter. Modeling of karst aquifer genesis: Influence of exchange flow. Water Resources Research, 39(10), 2003. http://dx.doi.org/10.1029/2003WR002218.

[4] S. Birk, R. Liedl, M. Sauter and G. Teutsch. Hydraulic boundary conditions as a controlling factor in karst genesis: A numerical modeling study on artesian conduit development in gypsum. Water Resources Research, 39(1):SBH 2-1SBH 2-14, 2003. http://dx.doi.org/10.1029/2002WR001308.

[5] Y. Cao, M. Gunzburger, F. Hua and X. Wang. Analysis and finite element approximation of a coupled, continuum pipe-flow/Darcy model for flow in porous media with embedded conduits. Numerical Methods for Partial Differential Equations, 27(5):1242-1252, 2011. http://dx.doi.org/10.1002/num.20579. 
[6] N. Chen, M. Gunzburger, B. Hu, X. Wang and C. Woodruff. Calibrating the exchange coefficient in the modified coupled continuum pipe-flow model for flows in karst aquifers. Journal of Hydrology, 414-415:294-301, 2012. http://dx.doi.org/10.1016/j.jhydrol.2011.11.001.

[7] S. Chen and D. Shi. Accuracy analysis for quasi-Wilson element. Acta Mathematica Scientia, 20(1):44-48, 2000.

[8] S. Chen, D. Shi and Y. Zhao. Anisotropic interpolation and quasi-Wilson element for narrow quadrilateral meshes. IMA Journal of Numerical Analysis, 24(1):7795, 2004. http://dx.doi.org/10.1093/imanum/24.1.77.

[9] Z. Chen, K. An, Y. Liu and W. Chen. Adjoint method for an inverse problem of CCPF model. Chinese Annals of Mathematics, Series B, 35(3):337-354, 2014. http://dx.doi.org/10.1007/s11401-014-0837-9.

[10] S. Lei. An analytical solution for steady flow into a tunnel. Ground Water, 37(1):23-26, 1999. http://dx.doi.org/10.1111/j.1745-6584.1999.tb00953.x.

[11] R. Liedl, M. Sauter, D. Hückinghaus, T. Clemens and G. Teutsch. Simulation of the development of Karst aquifers using a coupled continuum pipe flow model. Water Resources Research, 39(3), 2003. http://dx.doi.org/10.1029/2001WR001206.

[12] W. Liu, Z. Wang and J. Li. Anisotropic finite element approximation for a coupled continuum pipe-flow/Darcy model in Karst aquifers. Computers \& Mathematics with Applications, 68(3):86-100, 2014. http://dx.doi.org/10.1016/j.camwa.2014.05.009.

[13] W. Liu, Q. Zhao, X. Li and J. Li. Anisotropic Wilson element with conforming finite element approximation for a coupled continuum pipe-flow/Darcy model in Karst aquifers. Mathematical Methods in the Applied Sciences, 38(17):40244037, 2015. http://dx.doi.org/10.1002/mma.3341.

[14] V. Martin, J. Jaffré and J.E. Roberts. Modeling fractures and barriers as interfaces for flow in porous media. SIAM Journal on Scientific Computing, 26(5):1667-1691, 2005. http://dx.doi.org/10.1137/S1064827503429363.

[15] D. Shi and S. Chen. A kind of improved Wilson arbitrary quadrilateral elements. Numerical Mathematics A Journal of Chinese Universities (in Chinese), 16(2):161-167, 1994.

[16] D. Shi and S. Chen. A class of six-parameter nonconforming arbitrary convex quadrilateral elements. Appl. Math. J. Chin. Univ. (in Chinese), 11(2):231-238, 1996.

[17] Z.-C. Shi and S. Chen. Analysis of a nine degree plate bending element of shecht. Chinese Journal Numerical Mathematics Applications, 4:73-79, 1989.

[18] Z.-C. Shi, B. Jiang and W. Xue. A new superconvergence property of Wilson nonconforming finite element. Numerische Mathematik, 78(2):259-268, 1997. http://dx.doi.org/10.1007/s002110050312.

[19] X. Wang. On the coupled continuum pipe flow model (CCPF) for flows in Karst aquifer. Discrete and Continuous Dynamical Systems - Series B, 13(2):489-501, 2009. http://dx.doi.org/10.3934/dcdsb.2010.13.489.

[20] X. Wu, P. Kügler and S. Lu. Identification of the exchange coefficient from indirect data for a coupled continuum pipe-flow model. Chinese Annals of Mathematics, Series B, 35(3):483-500, 2014. http://dx.doi.org/10.1007/s11401-0140830-3. 